\title{
An update on the histological subtypes of hepatocellular carcinoma
}

\author{
Regina Cheuk-Lam Lo ${ }^{1,2}$ \\ 'Department of Pathology, The University of Hong Kong, Hong Kong, China. \\ ${ }^{2}$ State Key Laboratory of Liver Research, The University of Hong Kong, Hong Kong, China.
}

Correspondence to: Regina Cheuk-Lam Lo, MBChB, FRCPA, FHKAM (Pathology), Department of Pathology, The University of Hong Kong, Queen Mary Hospital, Pokfulam, Hong Kong SAR, China. E-mail: reginalo@pathology.hku.hk

How to cite this article: Lo RCL. An update on the histological subtypes of hepatocellular carcinoma. Hepatoma Res 2019;5:41. http://dx.doi.org/10.20517/2394-5079.2019.021

Received: 18 Oct 2019 First Decision: 31 Oct 2019 Revised: 7 Nov 2019 Accepted: 11 Nov 2019 Published: 15 Nov 2019

Science Editor: Dalbir Sandhu Copy Editor: Jing-Wen Zhang Production Editor: Tian Zhang

\begin{abstract}
Hepatocellular carcinoma (HCC) is one of the top-ranking cancers worldwide and in Southeast Asia. The high propensity for tumor recurrence, distant metastasis and chemoresistance remain major hurdles in the treatment of HCC. With advances on genetics and genomics research, molecular targeted therapies are emerging as a hope for better disease control. On the histological perspective, microscopic review of clinical samples has led to subclassification of HCC and establishment of new entities. In this review, latest understanding on macrotrabecular-massive HCC, steatohepatitic HCC, lymphocyte-rich HCC, scirrhous HCC, fibrolamellar carcinoma and combined hepatocellular-cholangiocarcinoma will be discussed, emphasizing on the clinical relevance of these pathological entities. Further delineation of the histological, immunohistochemical, molecular and biological phenotypes of primary liver cancer would to further enhance an integrated morphological-molecular classification that better predicts clinical outcome and guides clinical management.
\end{abstract}

Keywords: Liver cancer, subtype, histology

\section{INTRODUCTION}

Hepatocellular carcinoma (HCC) is the most common primary liver cancer. Microscopically, it is characterized by thickened cell plates, malignant tumor cell cytology, capillarization of sinusoids and evidence of invasion. Histological evaluation of HCC specimens plays a key role in tumor staging, and

cc) (i) (c) The Author(s) 2019. Open Access This article is licensed under a Creative Commons Attribution 4.0 International License (https://creativecommons.org/licenses/by/4.0/), which permits unrestricted use, sharing, adaptation, distribution and reproduction in any medium or format, for any purpose, even commercially, as long as you give appropriate credit to the original author(s) and the source, provide a link to the Creative Commons license, and indicate if changes were made. 
in distinguishing HCC from its precursor lesions or other liver nodules. With reference to the multi-step process of hepatocarcinogenesis which could also be observed histologically, some classification systems have been proposed for HCC. For instance, the terms "early HCC" and "progressed HCC" have been defined based on the size and differentiation of tumor ${ }^{[1]}$. In recent years, further investigations have been carried out on the subtyping of HCC specifically referencing the morphological characteristics of tumor cells. The significance of these subtypes was further substantiated by their clinical relevance and the genetic makeup. In the latest 5th edition of the World Health Organization (WHO) Classification of Digestive System Tumors, several histological subtypes were described ${ }^{[2]}$. In this review, we will focus on elaborating the recent understanding on 5 subtypes: macrotrabecular-massive HCC (MTM-HCC), steatohepatitic HCC (SH-HCC), lymphocyte-rich HCC, scirrhous HCC and fibrolamellar carcinoma (FLC). In addition, an update on the entity combined hepatocellular-cholangiocarcinoma (cHCC-CCA) will be discussed.

\section{MTM-HCC}

Published in 2018, histological review of clinical samples archive led to the identification of a novel and distinct subtype of HCC defined by the histological features of tumor cells - MTM-HCC. It is defined as the presence of macrotrabeculae of more than 6 cells thick in $>50 \%$ tumor, and was identified in $16 \%$ on average in 2 large cohorts comprising 237 surgical resection samples and 284 biopsy samples ${ }^{[3]}$. And this subtype was statistically associated with aggressive parameters including tumor size, alpha-fetoprotein (AFP) levels, satellite nodules and vascular invasion. Besides, it was an independent prognosticator for early recurrence (within 2 years) and overall recurrence ${ }^{[3]}$. The prognostic significance was further validated by other group ${ }^{[4]}$. In another study by Jeon et al. ${ }^{[5]}$, MTM-HCC, as defined by $>30 \%$ of macrotrabecular pattern, was associated with large tumor, hepatitis B virus infection, and less frequent cirrhosis. This subtype was also found to be associated with higher tumor grade, tumor stage, higher AFP level, and a worse recurrence-free survival.

In addition, investigations were carried out to identify specific radiological, immunohistochemical, and genetic features of this entity. Radiologically, MTM-HCC was reported to preferentially demonstrate irregular rim-like arterial phase enhancement on gadoxetate-enhanced magnetic resonance imaging ${ }^{[6]}$. Extending from their initial observation, Calderaro et al. ${ }^{[7]}$ attempted to identify potential biomarkers for this entity. On analysis of the TCGA dataset, endothelial-specific molecule 1 (ESM1) was identified and validated as a biomarker for MTM-HCC. In addition, angiotensin 2 and VEGFA overexpression was observed in MTM subtype ${ }^{[8]}$. Recent studies also shed light on the genetic composition of MTM-HCC, which was found to be related to cell cycle activation, chromosomal instability, the G3 transcriptomic subgroup $^{[8]}$ according to Boyault et al. ${ }^{[9]}$ and TP53 mutation ${ }^{[8]}$.

\section{SH-HCC}

The steatohepatitic subtype was first described by Salomao et al. ${ }^{[10]}$. It is characterized by prominent steatotic changes in the tumor cells namely fat accumulation, ballooning degeneration, presence MalloryDenk bodies and peri-cellular fibrosis. In a study examining HCV-related liver explants, SH-HCC was identified in $35.5 \%$ of the cohort ${ }^{[10]}$. According to a follow-up study by the same group in 2012 , SH-HCC constituted $14 \%$ of HCC explants ${ }^{[11]}$. A more recent paper reported a diagnosis of SH-HCC in around $20 \%$ of 96 HCC cases reviewed, and made an observation that SH-HCC was not associated with cirrhosis ${ }^{[12]}$. It was noted that around 60\% (14 of 22) SH-HCCs was associated with one more known risk factor for non-alcoholic fatty liver disease (NAFLD) ${ }^{[10]}$. The association with and NAFLD and metabolic syndrome was consolidated in other studies ${ }^{[13,14]}$. While most studies suggested a link of fatty liver disease with this subtype, in 2015 Yeh et al ${ }^{[15]}$ looked at a series of SH-HCC and identified a group of patients without any underlying causes for metabolic disease. 
Regarding correlation with other histological tumor parameters, there was a lack of microsatellite nodules or microvascular invasion in $\mathrm{SH}-\mathrm{HCC}^{[8]}$. On the immunohistochemical phenotype, $\mathrm{C}$ reactive protein (CRP) expression was frequent ${ }^{[8]}$. Immunohistochemical expression of serum amyloid A and CRP was significantly higher in this subtype than conventional HCC as revealed by another study ${ }^{[16]}$. It was also found that the cancer-associated fibroblasts in SH-HCC more frequently expressed senescence-associated secretory phenotype by immunohistochemical staining ${ }^{[14]}$. On genetic and genomic levels, SH-HCC was shown to associate with IL6/JAK/STAT pathway activation, as well as wild type $C_{T N N B}{ }^{[8,17]}$ and $T P 53^{[8]}$. By multivariate modeling, it was shown to be related to the $S 1$ subclass ${ }^{[12]}$ according to Hoshida et al. ${ }^{[18]}$.

\section{LYMPHOCYTE-RICH HCC}

Previously known as lymphoepithelioma-like HCC, lymphocyte-rich HCC is characterized by an immune-rich stroma ${ }^{[19,20]}$. Wada et al. ${ }^{[21]}$ defined this subtype by the presence of more than 100 tumorinfiltrating lymphocytes in 10 high-power fields. Despite a difference in immune cells infiltration, immunohistochemically the tumor cells express epithelial markers and HepPar-1 ${ }^{[22-27]}$. The tumorinfiltrating lymphocytes were largely composed of $\mathrm{CD} 3+\mathrm{T}$ cells ${ }^{[19-29]}$. In contrast to lymphoepitheliomalike carcinoma originating in the nasopharynx, vast majority of lymphocyte-rich HCC were EBER negative ${ }^{[19,22,23,26,28,30]}$. In 2017 , Labgaa et al. ${ }^{[31]}$ published a comprehensive review on a total of 66 lymphocyterich HCC cases. In this report, $64 \%$ patients were male and liver cirrhosis was present in $46 \%$. While a few studies demonstrated a trend of better survival with this subtype ${ }^{[19-21,28]}$, the prognostic significance of this subtype remains to be clarified due to its rarity. The genomic landscape of 12 lymphocyte-rich HCC was determined by whole-exome sequencing in a recent report ${ }^{[32]}$. Mutations of CTNNB1, AXIN1, APC, $\mathrm{NOTCH}_{1}$ and $\mathrm{NOTCH} 2$ were less frequently observed in lymphocyte-rich HCC than conventional HCC. Since activation of Wnt/beta-catenin pathway was correlated with poorer clinical response to immune checkpoint inhibitors ${ }^{[33]}$, lymphocyte-rich HCC is possibly more susceptible to immunotherapies. The potential significance in terms of treatment response was in line with in a recent study examining the immunohistochemical expression in $217 \mathrm{HCCs}$, that a high programmed death-ligand 1 expression was correlated with the lymphocyte-rich subtype ${ }^{[34]}$.

\section{SCIRRHOUS HCC}

Scirrhous HCC shows peculiar histology with small oval cells arranged in nests or trabecular among an abundant fibrous stroma ${ }^{[35]}$. It comprises $0.19 \%$ of all HCC from the National Cancer Database from 2004$2015^{[36]}$. The survival outcome for this subtype remains to be further delineated. Overall survival of patients was found comparable with non-scirrhous HCC in some studies ${ }^{[36,37]}$, while both better ${ }^{[38-40]}$ and worse ${ }^{[35]}$ survival outcomes were also reported. Furthermore, scirrhous HCC was associated with less frequent HBV infection, lower serum AFP level and less liver cirrhosis when compared with conventional HCC ${ }^{[37]}$. Radiologically, scirrhous HCC was reported to show distinct computed tomography (CT) scan features including presence of washout areas ${ }^{[41]}$. Immunohistochemical analyses revealed expression of stem/ progenitor markers in scirrhous HCC; and gene expression profiling highlighted a TGF- $\beta$ signature ${ }^{[35]}$.

\section{FLC}

FLC was first introduced in $1956^{[42]}$ illustrating a primary liver cancer displaying characteristic large eosinophilic tumor cells with prominent nucleoli and pale bodies, and the prominent fibrotic bands traversing the tumor cells in lamellae. The latter feature led to the coining of its nomenclature ${ }^{[43]}$. FLC occurs more often in young adults with a mean age of diagnosis at 25 years ${ }^{[43-45]}$. FLC express CK7 and HepPar-1 immunohistochemical staining ${ }^{[46]}$. From a nationwide study published in 2014 using the SEER data base, the incidence of FLC was $1 \%$ among 7225 patients $^{[47]}$. In the same study, it was reported that patients tend to be younger, female, and associated with longer overall survival on univariate analysis. In 2014, it was reported that a chimeric transcript was identified, which was further found to be due to a 
deletion in chromosome 19 detected by whole genome sequencing, which in turn leads to the generation of the DNAJB1-PRKACA chimeric protein, with the kinase activity is retained in the latter component ${ }^{[48]}$. This discovery is significant since it provides a pathognomonic genetic feature for this subtype. The tumorigenicity of the fusion transcript was validated by in vivo mouse model with hydrodynamic tail vein injection of Crispr/cas9 generated DNAJB1-PRKACA vector ${ }^{[49]}$. Subsequent study revealed an interaction between the fusion kinase and $\beta$-catenin ${ }^{[50]}$, suggesting a contributory role of the fusion protein and $\beta$-catenin in the pathogenesis of FLC. In addition, analysis of clinical samples suggested the recruitment of heat shock protein 70 by the fusion enzyme and further in phosphoproteomic profiling using cell line models highlights the activation of ERK signaling in DNAJ-PKAC cells ${ }^{[51]}$.

\section{CHCC-CCA}

cHCC-CCA is defined as a primary liver cancer showing unequivocal presence of both hepatocytic and cholangiocytic differentiation in the same tumor ${ }^{[2,52]}$. The 2 components histologically can either be juxtaposed with or intermingled with each other. There is no definite cutoff value as to the minimal proportion of each component present in a tumor to render a diagnosis of cHCC-CCA. In this type of liver cancer, small uniform epithelial cells with scanty cytoplasm and showing CK19, EpCAM, CD56, CD117 or CD133 expression has been observed ${ }^{[2]}$. The radiological feature with CT scan/magnetic resonance imaging was reviewed by a French group ${ }^{[53]}$. In the study, a mixed pattern comprising HCC, intrahepatic cholangiocarcinoma and atypical radiological pattern was observed in CHCC-CCA; and this mixed pattern showed a sensitivity of $48 \%$ and a specificity of $81 \%$. Protein expression for of diagnostic purpose of cHCCCCA has been investigated, and malic enzyme 1 (ME1) was proposed as a potential immunohistochemical marker for cHCC-CCA, in which $77 \%$ express $\mathrm{ME}^{[54]}$.

Previous study demonstrated an intermediate clinical outcome of cHCC-CCA between HCC and intrahepatic cholangiocarcinoma (iCCA), when overall survival after resection, disease-free survival after resection, and overall survival after liver transplantation were considered ${ }^{[55]}$. A more recent study comprising $250 \mathrm{cHCC}-\mathrm{CCA}$ in the training cohort and 99 cases in the validation cohort demonstrated that the 1-, 2 and 3-year overall survival was $67.7 \%, 46.8 \%$ and $37.9 \%$ respectively; and the 1-, 2 and 3-year cancer-specific survival was $73.1 \%, 52.0 \%$ and $43.0 \%$, respectively ${ }^{[56]}$. At times of recurrence or metastasis, as reported by He et al. ${ }^{[56]}$, the heterogeneity tends to be retained rendering the clinical behavior of cHCCCCA recurrence is largely unpredictable ${ }^{[57]}$.

Despite the deviation in clinical outcome, a study on 20 cHCC-CCA samples by capture-based nextgeneration sequencing revealed similar genomic profiles to conventional HCC. Recurrent alterations in TERT, TP53, cell cycle genes, receptor tyrosine kinase/Ras/PI3K pathway genes, chromatin regulators, etc., were identified in $\mathrm{CHCC}-\mathrm{CCA}$, while IDH1, IDH2, FGFR2 or BAP1 mutations were absent ${ }^{[58]}$. On a side note, genomic and genetic profiling of cholangiolocellular carcinoma, which was previously classified as a subtype of CHCC-CCA in the 4th edition of WHO Classification of Digestive Tumors ${ }^{[59]}$, showed that this entity was likely biliary tract origin featuring NCAM expression, chromosomal stability and TGF- $\beta$ activation ${ }^{[60]}$. Consistent findings were reported by Balitzer et al. ${ }^{[61]}$. By comparing immunohistochemical expressions, mutational profiles and copy number variation patterns, cholangiolocellular carcinoma was shown to display a highly similar pattern with iCCA, suggesting that the former should instead be classified as a form of well differentiated iCCA.

\section{FUTURE PERSPECTIVES}

In this review, latest understanding on 5 HCC subtypes and the distinct entity cHCC-CCA were discussed. These entities in common demonstrate peculiar pathognomonic histological features. Among these entities, MTM-HCC, lymphocyte-rich HCC and cHCC-CCA are known carry potential prognostic significance. 
In addition, lymphocyte-rich HCC may represent a subtype showing relatively favorable response to immunotherapy. SH-HCC may represent a spectrum of HCC arising from specific etiology. Further delineation of the genetic and genomic signatures of FLC and cHCC-CCA may provide insights on the cell of origin and pathogenesis of primary liver cancer.

Apart from defining specific subtypes, some histological features in HCC were found to be closely related to certain genetic alterations. For instance, well differentiated tumors with pseudoacinar pattern, tumor cell cholestasis and lack of immune cell infiltration were associated with CTNNB1 mutations ${ }^{[8,62]}$. Calderaro et al ${ }^{[63]}$ summarized a histological-molecular correlation of liver cancer. In this review, the molecular subclasses ${ }^{[9,18]}$ and genetic alterations of histological subtypes including MTM-HCC, SH-HCC, scirrhous HCC, lymphocyterich HCC were discussed. Besides, the immune microenvironment of 158 HCC cases was recently characterized by Kurebayashi et al. ${ }^{[64]}$ using multiplex immunohistochemistry. The accumulating body of information, together with integrated analyses of the expression profiles of HCC at transcriptomic, genomic and proteomic levels may facilitate formulating a classification system of clinical relevance.

\section{DECLARATIONS}

\section{Authors' contributions}

The author contributed solely to the article.

\section{Availability of data and materials}

Not applicable.

\section{Financial support and sponsorship}

Clinical Research Fellowship Scheme (General Research Fund:17126216), Research Grants Council, Hong Kong.

\section{Conflicts of interest}

The author declared that there are no conflicts of interest.

\section{Ethical approval and consent to participate}

Not applicable.

\section{Consent for publication}

Not applicable.

\section{Copyright}

(c) The Author(s) 2019.

\section{REFERENCES}

1. Roncalli M, Park YN, Di Tommaso L. Histopathological classification of hepatocellular carcinoma. Dig Liver Dis 2010;42 supp$\mathrm{S} 3: \mathrm{S} 228-34$.

2. Bosman FT, Carneiro F, Hruban RH. WHO classification of tumours of the digestive system. 5th ed. Geneva: International agency for Research on Cancer; 2019. p. 229.

3. Ziol M, Pote N, Amaddeo G, Laurent A, Nault JC, et al. Macrotrabecular-massive hepatocellular carcinoma: a distinctive histological subtype with clinical relevance. Hepatology 2018;68:103-12.

4. Okabe H, Yoshizumi T, Yamashita YI, Imai K, Hayashi H, et al. Histological architectural classification determines recurrence pattern and prognosis after curative hepatectomy in patients with hepatocellular carcinoma. PLoS One 2018;13:e0203856.

5. Jeon Y, Benedict M, Taddei T, Jain D, Zhang X. Macrotrabecular hepatocellular carcinoma: an aggressive subtype of hepatocellular carcinoma. Am J Surg Pathol 2019;43:943-8.

6. Rhee H, An C, Kim HY, Yoo JE, Park YN, et al. Hepatocellular carcinoma with irregular rim-like arterial phase hyperenhancement: more aggressive pathologic features. Liver Cancer 2019;8:24-40.

7. Calderaro J, Meunier L, Nguyen CT, Boubaya M, Caruso S, et al. ESM1 as a marker of macrotrabecular-massive hepatocellular 
carcinoma. Clin Cancer Res 2019;25:5859-65.

8. Calderaro J, Couchy G, Imbeaud S, Amaddeo G, Letouze E, et al. Histological subtypes of hepatocellular carcinoma are related to gene mutations and molecular tumour classification. J Hepatol 2017;67:727-38.

9. Boyault S, Rickman DS, de Reynies A, Balabaud C, Rebouissou S, et al. Transcriptome classification of HCC is related to gene alterations and to new therapeutic targets. Hepatology 2007;45:42-52.

10. Salomao M, Yu WM, Brown RS Jr, Emond JC, Lefkowitch JH. Steatohepatitic hepatocellular carcinoma (SH-HCC): a distinctive histological variant of HCC in hepatitis C virus-related cirrhosis with associated NAFLD/NASH. Am J Surg Pathol 2010;34:1630-6.

11. Salomao M, Remotti H, Vaughan R, Siegel AB, Lefkowitch JH, et al. The steatohepatitic variant of hepatocellular carcinoma and its association with underlying steatohepatitis. Hum Pathol 2012;43:737-46.

12. Tan PS, Nakagawa S, Goossens N, Venkatesh A, Huang T, et al. Clinicopathological indices to predict hepatocellular carcinoma molecular classification. Liver Int 2016;36:108-18.

13. Jain D, Nayak NC, Kumaran V, Saigal S. Steatohepatitic hepatocellular carcinoma, a morphologic indicator of associated metabolic risk factors: a study from India. Arch Pathol Lab Med 2013;137:961-6.

14. Lee JS, Yoo JE, Kim H, Rhee H, Koh MJ, et al. Tumor stroma with senescence-associated secretory phenotype in steatohepatitic hepatocellular carcinoma. PLoS One 2017;12:e0171922.

15. Yeh MM, Liu Y, Torbenson M. Steatohepatitic variant of hepatocellular carcinoma in the absence of metabolic syndrome or background steatosis: a clinical, pathological, and genetic study. Hum Pathol 2015;46:1769-75.

16. Taniai M, Hashimoto E, Tobari M, Kodama K, Tokushige K, et al. Clinicopathological investigation of steatohepatitic hepatocellular carcinoma: a multicenter study using immunohistochemical analysis of adenoma-related markers. Hepatol Res 2018;48:947-55.

17. Ando S, Shibahara J, Hayashi A, Fukayama M. $\beta$-catenin alteration is rare in hepatocellular carcinoma with steatohepatitic features: immunohistochemical and mutational study. Virchows Arch 2015;467:535-42.

18. Hoshida Y, Nijman SM, Kobayashi M, Chan JA, Brunet JP, et al. Integrative transcriptome analysis reveals common molecular subclasses of human hepatocellular carcinoma. Cancer Res 2009;69:7385-92.

19. Chan AW, Tong JH, Pan Y, Chan SL, Wong GL, et al. Lymphoepithelioma-like hepatocellular carcinoma: an uncommon variant of hepatocellular carcinoma with favorable outcome. Am J Surg Pathol 2015;39:304-12.

20. Patel KR, Liu TC, Vaccharajani N, Chapman WC, Brunt EM. Characterization of inflammatory (lymphoepithelioma-like) hepatocellular carcinoma: a study of 8 cases. Arch Pathol Lab Med 2014;138:1193-202.

21. Wada Y, Nakashima O, Kutami R, Yamamoto O, Kojiro M. Clinicopathological study on hepatocellular carcinoma with lymphocytic infiltration. Hepatology 1998;27:407-14.

22. Chen CJ, Jeng LB, Huang SF. Lymphoepithelioma-like hepatocellular carcinoma. Chang Gung Med J 2007;30:172-7.

23. Cacciato Insilla A, Faviana P, Pollina LE, De Simone P, Coletti L, et al. Lymphoepithelioma-like hepatocellular carcinoma: case report and review of the literature. World J Gastroenterol 2015;21:10468-74.

24. Shinoda M, Kadota Y, Tsujikawa H, Masugi Y, Itano O, et al. Lymphoepithelioma-like hepatocellular carcinoma: a case report and a review of the literature. World J Surg Oncol 2013;11:97.

25. Park HS, Jang KY, Kim YK, Cho BH, Moon WS. Hepatocellular carcinoma with massive lymphoid infiltration: a regressing phenomenon? Pathol Res Pract 2009;205:648-52.

26. Si MW, Thorson JA, Lauwers GY, DalCin P, Furman J. Hepatocellular lymphoepithelioma-like carcinoma associated with epstein barr virus: a hitherto unrecognized entity. Diagn Mol Pathol 2004;13:183-9.

27. Shirabe K, Matsumata T, Maeda T, Sadanaga N, Kuwano H, et al. A long-term surviving patient with hepatocellular carcinoma including lymphocytes infiltration - a clinicopathological study. Hepatogastroenterology 1995;42:996-1001.

28. Emile JF, Adam R, Sebagh M, Marchadier E, Falissard B, et al. Hepatocellular carcinoma with lymphoid stroma: a tumour with good prognosis after liver transplantation. Histopathology 2000;37:523-9.

29. Wei J, Liu Q, Wang C, Yu S. Lymphoepithelioma-like hepatocellular carcinoma without Epstein-Barr virus infection: a case report and a review of the literature. Indian J Pathol Microbiol 2015;58:550-3.

30. An SL, Liu LG, Zheng YL, Rong WQ, Wu F, et al. Primary lymphoepithelioma-like hepatocellular carcinoma: report of a locally advanced case and review of literature. Int J Clin Exp Pathol 2015;8:3282-7.

31. Labgaa I, Stueck A, Ward SC. Lymphoepithelioma-like carcinoma in liver. Am J Pathol 2017;187:1438-44.

32. Chan AW, Zhang Z, Chong CC, Tin EK, Chow C, et al. Genomic landscape of lymphoepithelioma-like hepatocellular carcinoma. J Pathol 2019;249:166-72.

33. Harding JJ, Nandakumar S, Armenia J, Khalil DN, Albano M, et al. Prospective genotyping of hepatocellular carcinoma: clinical implications of next-generation sequencing for matching patients to targeted and immune therapies. Clin Cancer Res 2019;25:2116-26.

34. Calderaro J, Rousseau B, Amaddeo G, Mercey M, Charpy C, et al. Programmed death ligand 1 expression in hepatocellular carcinoma: relationship with clinical and pathological features. Hepatology 2016;64:2038-46.

35. Seok JY, Na DC, Woo HG, Roncalli M, Kwon SM, et al. A fibrous stromal component in hepatocellular carcinoma reveals a cholangiocarcinoma-like gene expression trait and epithelial-mesenchymal transition. Hepatology 2012;55:1776-86.

36. Farooq A, Merath K, Paredes AZ, Wu L, Tsilimigras DI, et al. Outcomes of patients with scirrhous hepatocellular carcinoma: insights from the national cancer database. J Gastrointest Surg 2019; Epub ahead of print [DOI: 10.1007/s11605-019-04282-1]

37. Lee JH, Choi MS, Gwak GY, Lee JH, Koh KC, et al. Clinicopathologic characteristics and long-term prognosis of scirrhous hepatocellular carcinoma. Dig Dis Sci 2012;57:1698-707.

38. Fujii T, Zen Y, Harada K, Niwa H, Masuda S, et al. Participation of liver cancer stem/progenitor cells in tumorigenesis of scirrhous hepatocellular carcinoma - human and cell culture study. Hum Pathol 2008;39:1185-96.

39. Okamura N, Yoshida M, Shibuya A, Sugiura H, Okayasu I, et al. Cellular and stromal characteristics in the scirrhous hepatocellular carcinoma: comparison with hepatocellular carcinomas and intrahepatic cholangiocarcinomas. Pathol Int 2005;55:724-31. 
40. Sugiki T, Yamamoto M, Taka K, Nakano M. Specific characteristics of scirrhous hepatocellular carcinoma. Hepatogastroenterology 2009;56:1086-9.

41. Kim SH, Lim HK, Lee WJ, Choi D, Park CK. Scirrhous hepatocellular carcinoma: comparison with usual hepatocellular carcinoma based on CT-pathologic features and long-term results after curative resection. Eur J Radiol 2009;69:123-30.

42. Edmondson HA. Differential diagnosis of tumors and tumor-like lesions of liver in infancy and childhood. AMA J Dis Child 1956;91:168-86

43. Craig JR, Peters RL, Edmondson HA, Omata M. Fibrolamellar carcinoma of the liver: a tumor of adolescents and young adults with distinctive clinico-pathologic features. Cancer 1980;46:372-9.

44. Eggert T, McGlynn KA, Duffy A, Manns MP, Greten TF, et al. Fibrolamellar hepatocellular carcinoma in the USA, 2000-2010: a detailed report on frequency, treatment and outcome based on the surveillance, epidemiology, and end results database. United European Gastroenterol J 2013;1:351-7.

45. Torbenson M. Fibrolamellar carcinoma: 2012 update. Scientifica (Cairo) 2012;2012:743790.

46. Choi WT, Ramachandran R, Kakar S. Immunohistochemical approach for the diagnosis of a liver mass on small biopsy specimens. Hum Pathol 2017;63:1-13.

47. Mayo SC, Mavros MN, Nathan H, Cosgrove D, Herman JM, et al. Treatment and prognosis of patients with fibrolamellar hepatocellular carcinoma: a national perspective. J Am Coll Surg 2014;218:196-205.

48. Honeyman JN, Simon EP, Robine N, Chiaroni-Clarke R, Darcy DG, et al. Detection of a recurrent DNAJB1-PRKACA chimeric transcript in fibrolamellar hepatocellular carcinoma. Science 2014;343:1010-4.

49. Engelholm LH, Riaz A, Serra D, Dagnaes-Hansen F, Johansen JV, et al. CRISPR/Cas9 engineering of adult mouse liver demonstrates that the dnajb1-prkaca gene fusion is sufficient to induce tumors resembling fibrolamellar hepatocellular carcinoma. Gastroenterology 2017;153:1662-73.e10.

50. Kastenhuber ER, Lalazar G, Houlihan SL, Tschaharganeh DF, Baslan T, et al. DNAJB1-PRKACA fusion kinase interacts with betacatenin and the liver regenerative response to drive fibrolamellar hepatocellular carcinoma. Proc Natl Acad Sci U S A 2017;114:13076-84

51. Turnham RE, Smith FD, Kenerson HL, Omar MH, Golkowski M, et al. An acquired scaffolding function of the DNAJ-PKAc fusion contributes to oncogenic signaling in fibrolamellar carcinoma. Elife 2019;8:e44187.

52. Brunt E, Aishima S, Clavien PA, Fowler K, Goodman Z, et al. cHCC-CCA: consensus terminology for primary liver carcinomas with both hepatocytic and cholangiocytic differentation. Hepatology 2018;68:113-26.

53. Gigante E, Ronot M, Bertin C, Ciolina M, Bouattour M, et al. Combining imaging and tumor biopsy improves the diagnosis of combined hepatocellular-cholangiocarcinoma. Liver Int 2019.

54. Mihara Y, Akiba J, Ogasawara S, Kondo R, Fukushima H, et al. Malic enzyme 1 is a potential marker of combined hepatocellular cholangiocarcinoma, subtype with stem-cell features, intermediate-cell type. Hepatol Res 2019;49:1066-75.

55. Garancini M, Goffredo P, Pagni F, Romano F, Roman S, et al. Combined hepatocellular-cholangiocarcinoma: a population-level analysis of an uncommon primary liver tumor. Liver Transpl 2014;20:952-9.

56. He C, Zhang Y, Cai Z, Lin X. Competing risk analyses of overall survival and cancer-specific survival in patients with combined hepatocellular cholangiocarcinoma after surgery. BMC Cancer 2019;19:178.

57. De Vito C, Sarker D, Ross P, Heaton N, Quaglia A. Histological heterogeneity in primary and metastatic classic combined hepatocellular-cholangiocarcinoma: a case series. Virchows Arch 2017;471:619-29.

58. Joseph NM, Tsokos CG, Umetsu SE, Shain AH, Kelley RK, et al. Genomic profiling of combined hepatocellular-cholangiocarcinoma reveals similar genetics to hepatocellular carcinoma. J Pathol 2019;248:164-78.

59. Bosman FT, Carneiro F, Hruban RH. WHO classification of tumours of the digestive system. Fourth edition ed. International agency for research on cancer; 2010.

60. Moeini A, Sia D, Zhang Z, Camprecios G, Stueck A, et al. Mixed hepatocellular cholangiocarcinoma tumors: cholangiolocellular carcinoma is a distinct molecular entity. J Hepatol 2017;66:952-61.

61. Balitzer D, Joseph NM, Ferrell L, Shafizadeh N, Jain D, et al. Immunohistochemical and molecular features of cholangiolocellular carcinoma are similar to well-differentiated intrahepatic cholangiocarcinoma. Mod Pathol 2019;32:1486-94.

62. Audard V, Grimber G, Elie C, Radenen B, Audebourg A, et al. Cholestasis is a marker for hepatocellular carcinomas displaying betacatenin mutations. J Pathol 2007;212:345-52.

63. Calderaro J, Ziol M, Paradis V, Zucman-Rossi J. Molecular and histological correlations in liver cancer. J Hepatol 2019;71:616-30.

64. Kurebayashi Y, Ojima H, Tsujikawa H, Kubota N, Maehara J, et al. Landscape of immune microenvironment in hepatocellular carcinoma and its additional impact on histological and molecular classification. Hepatology 2018;68:1025-41. 